\title{
Surface core-level phonon broadening of $\mathrm{Li}(110)$
}

\author{
D. M. Riffe \\ Physics Department, Utah State University, Logan, Utah 84322-4415 \\ G. K. Wertheim \\ AT\&T Bell Laboratories, Murray Hill, New Jersey 07974 \\ (Received 9 July 1999)
}

\begin{abstract}
High-resolution core-level photoemission data from the $1 s$ level of $\operatorname{Li}(110)$ have been obtained between 77 and $280 \mathrm{~K}$. Analysis of the data reveals a significant difference in the zero-temperature phonon broadening between the bulk and surface atoms but only a small difference in the effective surface and bulk Debye temperatures. This latter result is in good agreement with an embedded-atom-method calculation of the bulk and surface Debye temperatures of Li. Implications of these results to surface core-level phonon broadening and surface lattice dynamics of the heavier alkali metals are discussed.
\end{abstract}

\section{INTRODUCTION}

The contribution of vibrational broadening to core-levelexcitation line shapes in solids has been discussed since at least the 1950s. ${ }^{1}$ Generally, the optical excitation of a core hole is coupled to vibrational modes of the lattice. This is due either to a change in excited-atom-neighboring-atom equilibrium positions (first-order coupling) or force constants (second-order coupling) upon excitation of a core hole. In a monatomic metal, where the core-electron excitation is coupled to a continuum of acoustic phonon modes, the result is a temperature-dependent Gaussian broadening of the electronic excitation spectrum. One of the first theories of vibrational broadening in a metal was due to Overhauser, ${ }^{2}$ whose linear-coupling, deformation-potential theory predicted a temperature-dependent phonon broadening given by

$$
G_{\mathrm{ph}}^{2}(T)=G_{\mathrm{ph}}^{2}(0)\left[1+8\left(\frac{T}{\theta_{D}}\right)^{4} \int_{0}^{\theta_{D} / T} \frac{x^{3}}{e^{x}-1} d x\right],
$$

where $G_{\mathrm{ph}}^{2}(T)$ is the square of the phonon width, $T$ is the temperature, and $\theta_{D}$ is the Debye temperature of the solid. Note that the expression in brackets is simply proportional to the internal energy in the phonons in the Debye model; $G_{\mathrm{ph}}^{2}(T)$ is thus proportional to $T$ for $T>\theta_{D}$. Subsequent to Overhauser's work, Hedin and Rosengren, ${ }^{3}$ with a pseudopotential theory, confirmed the temperature dependence of Overhauser's expression [Eq. (1)] and, further, expressed the zero-temperature phonon width as

$$
G_{\mathrm{ph}}^{2}(0)=\frac{C}{\theta_{D}},
$$

where $C$ is a constant that depends upon the derivative of the nearest-neighbor screened pseudopotential and the mass density of the solid. Other theoretical studies of temperaturedependent phonon broadening ${ }^{4,5}$ also exhibit the temperature dependence of Eq. (1). However, different calculations for the same metal often produce quite different values for the parameters $C$ and $\theta_{D} \cdot{ }^{2-5}$ Hence, the Debye temperature parameter $\theta_{D}$ in Eqs. (1) and (2) that governs phonon broaden- ing may not exactly match a Debye temperature that has been determined by other means.

Temperature-dependent core-level broadening in photoemission has been systematically investigated in several metallic systems. The earliest study, ${ }^{6}$ also on $\mathrm{Li}$, was insensitive to the surface layer of atoms, due to the large mean free path of the electrons photoemitted by $\mathrm{Al} K \alpha$ radiation ( $h \nu$ $=1487 \mathrm{eV}$ ). The results ${ }^{6,7}$ of that study are consistent with the temperature dependence exhibited by Eq. (1) with a bestfit Debye temperature of $477 \mathrm{~K}$. Other systematic temperature-dependent studies have all taken advantage of modern synchrotron sources operating in the vacuumultraviolet or soft-x-ray regions $(h \nu=20-150 \mathrm{eV})$. Core electrons photoemitted with photon energies in this range have much smaller mean free paths; the data are thus very surface sensitive and can, in principle, distinguish the broadening parameters for bulk and surface atoms. These more recent studies include measurements on $\mathrm{Na}(110),{ }^{8,9} \mathrm{~K}(110),{ }^{9}$ $\mathrm{Rb}(110),{ }^{9} \mathrm{Al}(100)$ and $\mathrm{Al}(111),{ }^{10} \mathrm{Yb}(110),{ }^{11,12} \mathrm{Lu}(0001),{ }^{12}$ and $\mathrm{Tb}(0001) .^{12}$ In the majority of these studies the temperature dependence of the photoemission linewidths is consistent with Eq. (1). Exceptions are exhibited by the hightemperature surface-atom phonon broadening of the heavier alkali metals $\mathrm{K}$ and $\mathrm{Rb}$, which appear to have a significant anharmonic contribution, ${ }^{9}$ and by $\mathrm{Al}$, which has a temperature-dependent broadening better described by purely second-order coupling. ${ }^{10}$ Additionally, in all of these cases, except for $\mathrm{Al}(111)$, the surface-atom Gaussian width is significantly larger than for atoms in the bulk.

There have also been a number of less systematic studies in which independent surface- and bulk-atom Gaussian widths have been measured. Systems that have been measured include $\mathrm{Cs}(110),{ }^{13} \mathrm{Be}(0001),{ }^{14} \mathrm{Ta}(110),{ }^{15} \mathrm{Ta}(100),{ }^{16}$ $\mathrm{W}(110),{ }^{17}$ and $\mathrm{W}(100) .{ }^{18}$ Four of these systems [Cs(110), $\mathrm{Be}(0001), \mathrm{Ta}(110)$, and $\mathrm{W}(100)$ ] exhibit surface-atom widths that are broader than that of the bulk atoms. However, for $\mathrm{W}(110)$ and $\mathrm{Ta}(100)$, the bulk and surface Gaussian widthsare equal to within experimental error.

In spite of this apparently large set of phonon-broadening data, no study has yet independently determined the broadening parameters $C$ and $\theta_{D}$ for both surface and bulk atoms. 
In order to do this, high-quality data must be obtained at temperatures sufficiently below the Debye-temperature parameter $\theta_{D}$ in Eq. (1). Otherwise the broadening is well described by the linear high-temperature limit

$$
G_{\mathrm{ph}}^{2}(T) \approx \frac{8 C T}{3 \theta_{D}^{2}},
$$

which precludes independent evaluations of $C$ and $\theta_{D}$. For example, in interpreting broadening data on the alkali ${ }^{8,9}$ and lanthanide $^{11,12}$ metals, the investigators assumed that the coupling constant $C$ is identical in bulk and at the surface, leading to the conclusion that the effective Debye temperature at the surface is much smaller than in the bulk. The larger surface Gaussian widths that are usually observed ${ }^{13-15,17}$ have also been taken as evidence for significantly smaller surface Debye temperatures. However, this simple picture of differences in bulk and surface broadening arising entirely from differences in $\theta_{D}$ is challenged by the results on $\mathrm{Al}(111), \mathrm{W}(110)$, and $\mathrm{Ta}(100)$, in which the surface-atom broadening is nearly the same as in the bulk.

In order to obtain data on a metallic system sufficient to independently extract $C$ and $\theta_{D}$ for bulk and surface atoms, we have investigated the temperature-dependent broadening of the $1 s$ level of $\operatorname{Li}(110)$ between 80 and $275 \mathrm{~K}$. Since the Debye temperature of $\mathrm{Li}$ is in the range of $350-400 \mathrm{~K},{ }^{19}$ the data have allowed us to determine both $C$ and $\theta_{D}$ in the bulk and surface. Our results indicate for $\operatorname{Li}(110)$ that the surface atom $C$ is approximately twice that of the bulk, while the effective surface Debye temperature is approximately 5\% less than in the bulk. In order to check the reasonableness of these results, we have used an embedded-atom-method (EAM) model $^{20}$ to calculate bulk and surface Debye temperatures from atomic mean-squared displacements. While the calculation produces $\theta_{D}$ 's that are significantly less than those extracted from the core-level broadening, the calculated surface values are approximately $10 \%$ less than in the bulk, in good agreement with the measurements. EAM modeling of $\mathrm{Na}$ and $\mathrm{K}$ suggests a similar ratio in surface and bulk Debye temperatures, implying a greater coupling constant $C$ at the (110) surface of these alkali metals as well.

\section{EXPERIMENTAL DETAILS}

Photoemission data were obtained on beamline U4A on the VUV ring at the National Light Source at Brookhaven National Laboratory. The synchrotron light was monochromatized with a 10-m torodial grating monochromator operated at $90 \mathrm{eV}$ with a resolution of $90 \mathrm{meV}$. The photoelectron spectra were collected with a $100-\mathrm{mm}$ Vacuum Science Workshop hemispherical analyzer operated with a pass energy of $5 \mathrm{eV}$ and resolution of $100 \mathrm{meV}$, yielding a total instrumental resolution of $134 \mathrm{meV}$. Ambient background pressure in the chamber was in the range of $(1-2) \times 10^{10}$ Torr, dominated by $\mathrm{H}_{2}$. As in the case of temperature-dependent $\mathrm{Na}(110)$ data, we observed no effect of background contamination on the spectra. ${ }^{8}$

The $\mathrm{Li}(110)$ samples were prepared by in situ condensation of metal vapor, obtained from an SAES Getters source

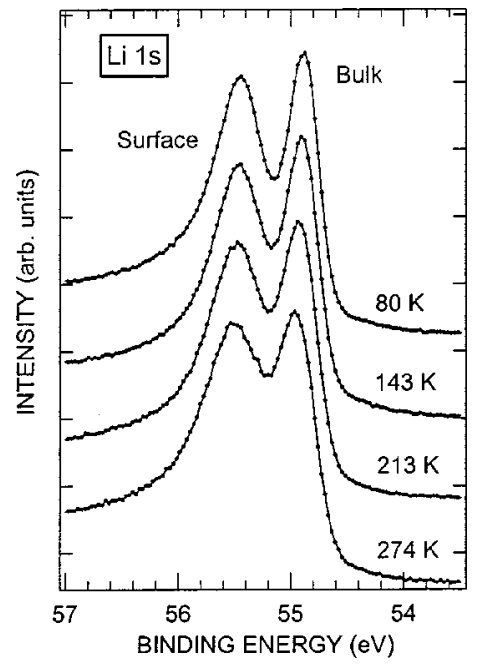

FIG. 1. Representative temperature-dependent Li(110) $1 s$ corelevel spectra obtained between 80 and $274 \mathrm{~K}$.

onto a liquid $\mathrm{N}_{2}$ cooled $\mathrm{Ni}(100)$ substrate. A total of 26 temperature-dependent spectra were then obtained over a 2-h period as the sample was allowed to warm up from $80 \mathrm{~K}$ towards room temperature. The temperature rise during any one scan was in the range of 5-10 K. As with other alkali metals deposited on $\mathrm{Ni}(100)$, the $\mathrm{Li}$ film is (110) oriented. The as-deposited film showed a rather diffuse low-energy electron-diffraction (LEED) pattern indicative of a multipledomain (110)-oriented surface. Annealing such a sample to approximately room temperature produces a single-domain (110) film with relatively sharp spots. In an experiment separate from the temperature-dependent measurements that are the focus of this paper, core-level spectra were obtained on both as-deposited and annealed-and-recooled samples to check for the effects of (static) inhomogeneous broadening on the Gaussian widths. From these data we estimate inhomogeneous broadening to contribute, at most, $6000 \mathrm{meV}^{2}$ to the total measured $G^{2}$. As will be seen below, this contribution is negligible and is henceforth ignored.

The thickness of the $\mathrm{Li}$ sample used in the temperaturedependent measurements was $15 \pm 1 \AA$, corresponding to six atomic layers. The thickness was obtained from the decrease in the intensity of the $\mathrm{Ni}$ substrate valence-band spectrum ${ }^{21}$ upon deposition of an identically prepared Li sample in conjunction with our previous determination of the electron mean free path in $\mathrm{Li}^{22}$ As discussed in more detail below, photoemission from the interfacial atomic layer of $\mathrm{Li}$ [i.e., the $\mathrm{Li}$ atoms bonded to the $\mathrm{Ni}(100)$ surface atoms], although strongly attenuated by inelastic scattering within the Li film, must be included in quantitative analysis of the Li core-level spectra.

\section{RESULTS AND ANALYSIS}

In Fig. 1 we display four of the 26 core-level spectra, obtained at $\sim 60 \mathrm{~K}$ intervals. The spectra exhibit two peaks: the higher-binding-energy peak is from atoms in the outermost atomic layer (surface) while the lower-binding-energy 


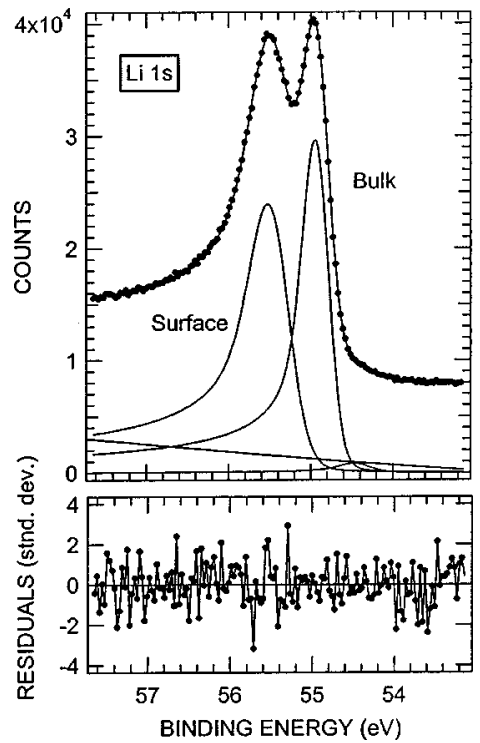

FIG. 2. Nonlinear least-squares analysis of a $\operatorname{Li}(110) 1 s$ corelevel spectrum. Surface, bulk, a small interface, and background contributions are displayed. The overall fit is the solid line through the data (solid circles). Residuals are shown in the bottom part of the figure.

feature is from atoms in layers 2-5 (bulk). Photoemission from interfacial $\mathrm{Li} \mathrm{(atomic} \mathrm{layer} \mathrm{1)} \mathrm{is} \mathrm{best} \mathrm{revealed} \mathrm{in} \mathrm{quan-}$ titative analysis of the data, although it does produce a small shoulder on the low-binding-energy side of the bulk peak. In addition to the temperature-dependent shift in binding energies, which has been previously discussed ${ }^{23}$ a temperaturedependent broadening in the core-level peaks from both the bulk and surface is clearly observed.

In order to extract the temperature-dependent Gaussian widths, the spectra were analyzed with nonlinear leastsquares curve fitting. In analyzing the data, we have used the standard line shape for metallic core levels: a DoniachSunjić line shape ${ }^{24}$ convolved with a Gaussian function. The resulting three line-shape parameters for each peak are (i) a Lorentzian width $\Gamma$, which is due to the finite lifetime of the excited core hole, (ii) a singularity index $\alpha$, which describes the long high-binding-energy tail of each peak and is related to the screening of the core hole by the conduction electrons, and (iii) the Gaussian width $G$, which is due to a combination of phonon broadening, instrumental resolution, and possibly inhomogeneous broadening. ${ }^{25}$ A smooth power-law function was found sufficient to model the background contribution to each spectrum.

A least-squares analysis of one of the data sets is shown in Fig. 2. In addition to the overall fit, the bulk, surface, and background contributions are separately shown. Also included is a much smaller peak at a binding energy of $\sim 54.4$ $\mathrm{eV}$. The size of this peak ( $2 \%$ of the total signal), its shift to lower binding energy (compared to the bulk), ${ }^{26}$ and its invariant intensity as a function of time identify it as from the interfacial Li layer (as opposed to a surface contamination feature). Although quite small, this peak is necessary in the analysis in order to remove nonstatistical fluctuations from the residuals, which are also displayed with the fit in Fig. 2.

Since the overall width of a core-level peak is determined by all three line-shape parameters $(\alpha, \Gamma$, and $G)$, and because

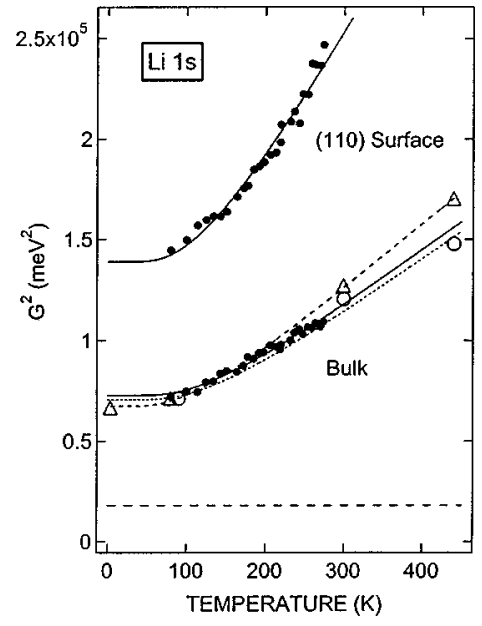

FIG. 3. Square of the Gaussian broadening vs temperature. Solid circles, this study; open circles, XPS study (Refs. 6 and 7); open triangles, AM theory (Ref. 5); lines: fits with Eq. (1); solid lines, this study; dotted line, XPS study; dashed line, AM theory.

there is significant overlap of the bulk and surface lines as well as the presence of the interface peak, accurate extraction of the phonon broadening is a nontrivial matter. Our approach has been to first determine as accurately as possible the bulk and surface singularity indices $(\alpha)$ and Lorentzian widths $(\Gamma)$, which are temperature independent. With those parameters constrained to their most reliable values, we then fit the complete set of data to extract the temperaturedependent Gaussian widths. To determine the singularity indices we fit the eight lowest temperature spectra (which have the highest intrinsic resolution due to the increasing Gaussian width versus temperature), extracting $\alpha_{\text {bulk }}=0.22 \pm 0.01$ and $\alpha_{\text {surface }}=0.30 \pm 0.01$. These values are identical to those that we previously obtained from independent data on $\operatorname{Li}(110){ }^{27}$ Determination of the Lorentzian widths was slightly more complicated. Since the interface peak falls directly on top of the low-binding-energy tail of the bulk peak, the bulk Lorentzian width and overall width of the interface peak have a significant correlation in the fitting process. From previous analysis of bulk-sensitive spectra, it was concluded that the bulk Lorentzian width is $40 \pm 20 \mathrm{meV} .{ }^{6,7} \mathrm{We}$ thus did several sets of analysis with the bulk Lorentzian width constrained to values in the range of $20-60 \mathrm{meV}$. Interestingly, throughout this range of bulk Lorentzian widths the fitted surface Lorentzian width was remarkably consistent at $45 \pm 10 \mathrm{meV}$. Given the close agreement between this surface value and the previously determined bulk value, we set both the surface and bulk $\Gamma$ 's to $45 \mathrm{meV}$. With the bulk and surface $\alpha$ 's and $\Gamma$ 's thus constrained, we extracted the Gaussian widths as a function of temperature. Note, however, that since the Lorentzian widths are much smaller than the Gaussian widths $(>250 \mathrm{meV})$, the extracted phonon widths are only weakly sensitive to variations of the Lorentzian widths within the range set by their statistical uncertainties.

In Fig. 3 we have plotted the square of the fitted Gaussian widths. The instrumental resolution is shown as the dashed line at $1.8 \times 10^{4} \mathrm{meV}^{2}$. Since Gaussian widths add in quadra- 
TABLE I. Phonon broadening parameters for Li $1 s$ core level. Parameters were obtained from fitting data with Eq. (1).

\begin{tabular}{llcccc}
\hline \hline Core level & \multicolumn{1}{c}{ Parameter } & Present study & $\mathrm{XPS}^{\mathrm{a}, \mathrm{c}}$ & AM theory $^{\mathrm{b}}$ & XAS $^{\mathrm{c}}$ \\
\hline Bulk & $\theta_{D}(\mathrm{~K})$ & $483 \pm 16$ & $477 \pm 120$ & $393 \pm 10$ & $278 \pm 71$ \\
& $G(0)(\mathrm{meV})$ & $231 \pm 2$ & $226 \pm 45$ & $221 \pm 3$ & $186 \pm 21$ \\
& $C\left(10^{-3} \mathrm{eV}^{3}\right)$ & $2.22 \pm 0.08$ & $2.10 \pm 1.00$ & $1.65 \pm 0.06$ & $0.83 \pm 0.28$ \\
Surface & $\theta_{D}(\mathrm{~K})$ & $452 \pm 17$ & & \\
& $G(0)(\mathrm{meV})$ & $346 \pm 4$ & & \\
& $C\left(10^{-3} \mathrm{eV}^{3}\right)$ & $4.66 \pm 0.21$ & & \\
& & & & \\
\hline \hline
\end{tabular}

${ }^{\mathrm{a}}$ Reference 6 .

${ }^{\mathrm{b}}$ Reference 5 .

${ }^{\mathrm{c}}$ Reference 7 .

ture, this dashed line can be thought of as the zero for the temperature-dependent broadening. At all temperatures $G_{\mathrm{ph}}^{2}$ for the surface is nearly double that for the bulk. Also shown in the figure, as open circles, are values for the bulk temperature-dependent width obtained from previous bulksensitive photoemission measurements. ${ }^{6,7}$ The good agreement between the previous bulk-sensitive results and our current results indicates that we have reliably determined the phonon broadening for both the bulk and the $\operatorname{Li}(110)$ surface atoms.

\section{DISCUSSION}

\section{A. Temperature dependence of bulk and surface Gaussian widths}

The temperature dependence of the experimental Gaussian widths (solid circles in Fig. 3) have been least-squaresanalyzed using Eq. (1) to determine best-fit values of $G_{\mathrm{ph}}(0), \theta_{D}$, and thus $C=G_{\mathrm{ph}}^{2}(0) \theta_{D}$. The least-squares fits, shown as the solid lines in Fig. 3, accurately characterize the data, indicating that the broadening in $\mathrm{Li}$ is consistent with linear coupling. As shown in Table I, the resulting value of $C$ for the surface is approximately two times larger than that for the bulk. In contrast, the Debye-temperature parameter is only $\sim 5 \%$ smaller at the surface than in the bulk. This is in marked contrast to previous interpretations of surface-atom phonon broadening in the alkali metals, where a much reduced surface $\theta_{D}$ was inferred from the much larger slope of the squared surface width for $T>\theta_{D} \cdot{ }^{8,9}$

We have also used Eq. (1) to analyze the temperaturedependent widths obtained in the earlier Li photoemission study. ${ }^{6,7}$ The fit is shown as the dotted line in Fig. 3. Summarized in Table I, the parameters extracted from the XPS study are quite close to those extracted from our present data. The much larger uncertainties associated with the XPSderived parameters are due to the fairly large error bars given for the phonon widths in that study, which were dominated by an estimated systematic uncertainty in the instrumental resolution. ${ }^{6}$ The excellent agreement between the present results and the XPS study suggests that the instrumentalresolution uncertainty in the XPS study may have been overestimated.

Theoretical values of the bulk-atom broadening from a linear-coupling, nonlinear-screening calculation of Almbladh and Morales (AM) (Ref. 5) are also shown in Fig. 3 as open triangles. This is the only theoretical calculation that comes reasonably close to the experimental results. As shown by $\mathrm{AM}$, inclusion of only linear screening in the pseudopotential produces widths that are only about half of the experimental values. Also of significance, AM have shown that secondorder coupling has a negligible effect on the core-hole widths, justifying the use of Eq. (1) to analyze the temperature dependence of the Gaussian widths. Fitting the AM theoretical values with Eq. (1) (dashed line through the theoretical data in Fig. 3), we extract a zero-temperature width of $221 \pm 3 \mathrm{meV}$, in very good agreement with our photoemission extracted values of $231 \pm 2 \mathrm{meV}$. The extracted Debye temperature of $393 \pm 10 \mathrm{~K}$, which is consistent with thermodynamic values of the Debye temperature, ${ }^{19}$ is $\sim 20 \%$ lower than our experimental value of $483 \pm 16 \mathrm{~K}$. The good agreement between the two experimental photoemission results indicates that systematic experimental error is likely not the cause of the discrepancy between the theoretical and experimental Debye-temperature parameters. A possible cause for the discrepancy may be that the AM theory underestimates the effects of second-order coupling (due to force-constant changes) on the phonon broadening. If the broadening is dominated by first-order coupling but has some contribution from second-order effects, then one may expect that Eq. (1) will sufficiently describe the data, but with a modified Debye temperature. Another possibility is that the photoemission process does not sample the lattice modes uniformly, favoring, on average, stiffer modes near the Brillouin-zone edges.

Phonon excitation also broadens x-ray-absorption edges, with a width that is theoretically identical to that obtained in photoemission. ${ }^{7}$ Temperature-dependent absorption-edge data of the $\mathrm{Li} 1 s$ level have been previously analyzed by Citrin and Wertheim. ${ }^{7}$ The extracted widths are not nearly as consistent with the theoretical values of AM as are the photoemission results. Fitting the absorption-edge-derived widths with Eq. (1) yields a zero-temperature width of 186 $\pm 21 \mathrm{meV}$ and a Debye temperature of $278 \pm 71 \mathrm{~K}$, both significantly lower that the parameters derived from AM's theoretical calculation or the photoemission studies. The differences between the XAS-derived parameters and those of the present study appear to be irreconcilable. We suggest that the differences may be related to an incomplete understanding of the $\mathrm{Li}$ absorption-edge line shape.

In prior work, an approximation to Eq. (1), 


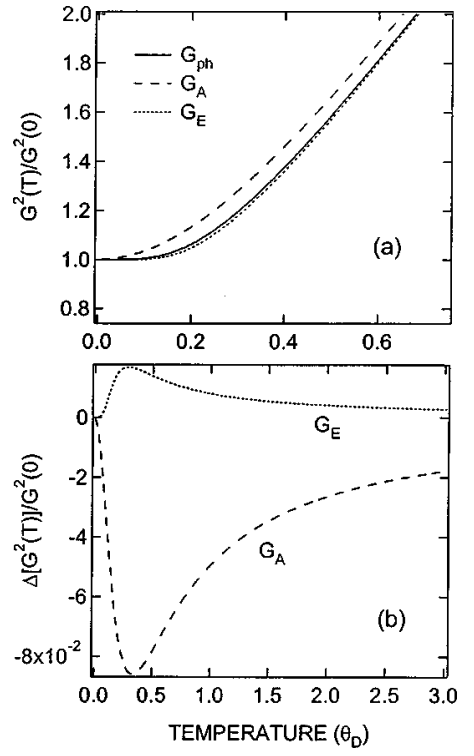

FIG. 4. Comparison of Debye model phonon broadening $G_{\mathrm{ph}}$ [Eq. (1)] with analytical phonon broadening approximations $G_{A}$ [Eq. (4)] and $G_{E}$ [Eq. (5)]. (a) Normalized squared Gaussian widths. (b) Differences in squared widths between Eq. (1) and the approximations.

$$
G_{A}^{2}(T)=G_{\mathrm{ph}}^{2}(0)\left[1+\left(\frac{8}{3} \frac{T}{\theta_{D}}\right)^{2}\right]^{1 / 2},
$$

was used to analyze temperature-dependent core-level phonon broadening. ${ }^{6,8,11,12}$ This approximation, which apparently originated with Overhauser, ${ }^{1}$ has the same hightemperature asymptote [Eq. (3)] and the same zerotemperature value $\left[G_{\mathrm{ph}}^{2}(0)\right]$ as Eq. (1). However, in the region $0<T / \theta_{D}<1$ the approximation does only a fair job of mimicking Eq. (1). This is illustrated in Fig. 4(a), which plots both Eq. (1) and the approximation [Eq. (4)] as solid and dashed lines, respectively. As shown in Fig. 4(b), which plots the difference between Eqs. (1) and (4), the error is as large as $\sim 8 \%$ at $T / \theta_{D} \approx 0.3$. As an illustration of the inaccuracy involved in using this approximation to analyze corelevel broadening, we have fit our temperature-dependent widths using Eq. (4). For the bulk width the fit yields $G_{\mathrm{ph}}(0)=222 \mathrm{meV}$ and $\theta_{D}=460 \mathrm{~K}$ compared to $231 \mathrm{meV}$ and $483 \mathrm{~K}$, respectively, from Eq. (1). For the surface data the discrepancy is even greater: $327 \mathrm{meV}$ and $414 \mathrm{~K}$ using Eq. (4) compared to $346 \mathrm{meV}$ and $452 \mathrm{~K}$ using Eq. (1).

If an analytical approximation to Eq. (1) is required, a much better equation that can be used is

$$
G_{E}^{2}(T)=G_{\mathrm{ph}}^{2}(0) \operatorname{coth}\left(\frac{3}{8} \frac{\theta_{D}}{T}\right) .
$$

This expression is simply the phonon broadening result for an Einstein oscillator ${ }^{6}$ with the oscillator energy $\hbar \omega_{0}$ replaced by $\frac{3}{4} k_{B} \theta_{D}$. This approximation and its difference with Eq. (1) are shown in parts (a) and (b) of Fig. 4, respectively. The much closer agreement with Eq. (1) is evident. In comparison with values obtained when using Eq. (1), the fitted values of $G_{\mathrm{ph}}(0)$ and $\theta_{D}$ from Eq. (5) vary by less than $2 \%$.

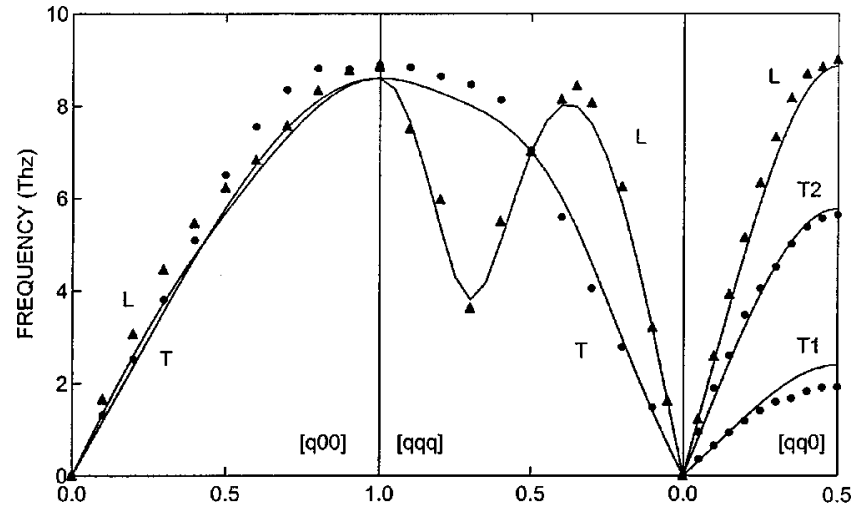

FIG. 5. Bulk phonon-dispersion curves for Li. Solid lines, results from WB EAM model; solid circles, experimental data (Ref. 26).

\section{B. EAM model calculations of Debye temperatures}

Since the temperature dependence of the core-electron broadening is intimately related to the Debye temperature, it would be appealing to have either theoretical calculations or other experimental measurements of the surface and bulk Debye temperatures of $\operatorname{Li}(110)$. However, as far as we know, there are no other experimental measurements or any theoretical calculations that provide either a value for the $\operatorname{Li}(110)$ surface Debye temperature or a relative comparison of $\mathrm{Li}$ bulk and (110) surface Debye temperatures. Therefore, in order to provide some insight into the (110) surface-layer vibrational dynamics of the alkali metals, we have used a semiempirical EAM model ${ }^{20}$ to calculate bulk and (110) surface Debye temperatures of $\mathrm{Li}, \mathrm{Na}$, and $\mathrm{K}$. The resulting values for $\mathrm{Na}$ and $\mathrm{K}$ impact the interpretation of previous experimental core-level broadening work ${ }^{8,9}$ on these metals.

The EAM model we have used is that of Wang and Boercker (WB). ${ }^{20}$ While there are several EAM models that have been developed for bcc metals, ${ }^{28}$ and are thus applicable to the alkali metals, this particular model was chosen since several experimental phonon frequencies are used as part of the input that determines the cohesive-energy parameters of the model. Following standard practice, we calculate phonon frequencies and polarizations by diagonalizing the dynamical matrix, which has contributions from both the two-body and embedded-atom terms in the cohesive energy. However, for the alkali metals, we have found that the embedded-atom term contributes negligibly to the lattice dynamics; for simplicity, the embedded-atom term has thus been omitted from our calculations. As shown in Fig. 5, the WB model does an excellent job of reproducing bulk phonon-dispersion curves for $\mathrm{Li}^{29}$ Note, for example, that the experimentally observed crossover of the transverse and longitudinal modes along the [100] direction is reproduced by the calculation. Experimental phonon-dispersion curves for $\mathrm{Na}$ (Ref. 30) and K (Ref. 31) are reproduced equally well by the WB model.

The Debye temperatures for the bulk were determined by considering formulas for mean-squared displacements in the high-temperature limit. In this limit the atomic mean-squared displacement $\left\langle u^{2}\right\rangle$ in the Debye model is related to the (angular) Debye frequency $\omega_{D}=k_{B} \theta_{D} / \hbar$ via 


$$
\left\langle u^{2}\right\rangle=\frac{9 k_{B} T}{M \omega_{D}^{2}},
$$

where $M$ is the mass of an atom in the solid. For a harmonic lattice, the mean-squared displacement at high temperatures is related to the (angular) phonon frequencies $\Omega_{n}$ via

$$
\left\langle u^{2}\right\rangle=\frac{k_{B} T}{N M} \sum_{n=1}^{3 N} \frac{1}{\Omega_{n}^{2}},
$$

where $3 N$ is the number of normal modes in the solid. Setting Eqs. (6) and (7) equal, we can thus define the bulk Debye temperature in terms of the phonon frequencies as

$$
\theta_{D, \text { bulk }}=\frac{3 \hbar}{k_{B}}\left(\frac{1}{N} \sum_{n=1}^{3 N} \frac{1}{\Omega_{n}^{2}}\right)^{-1 / 2} .
$$

Using Eq. (8) with phonon frequencies calculated at 215 evenly spaced points in the Brillouin zone, we have obtained the bulk Debye temperatures shown in Table II. These values compare favorably with experimentally determined values from specific-heat measurements. ${ }^{19}$

Calculation of surface Debye temperatures is more involved than for the bulk. Since surface atoms are no longer in an environment with cubic symmetry, the mean-squared displacements along the $x, y$, and $z$ directions are no longer equal. This allows one to define directional Debye temperatures. In analogy with Eq. (6), a directional Debye frequency $\omega_{D, x}$ can be defined in terms of the mean-squared displacement along a particular direction (in this case the $x$ direction) as

$$
\left\langle x^{2}\right\rangle=\frac{3 k_{B} T}{M \omega_{D, x}^{2}} .
$$

For a harmonic lattice, the high-temperature mean-squared displacement along the same direction is given by

$$
\left\langle x^{2}\right\rangle=\frac{k_{B} T}{N M} \sum_{n=1}^{3 N} \hat{x}_{n}^{2} \frac{1}{\Omega_{n}^{2}},
$$

where $\hat{x}_{n}$ is the projection of the normalized polarization vector (at the atom of interest) onto the $x$ direction. By equating Eqs. (9) and (10), the directional Debye temperature can be defined in terms of the vibrational modes of the solid. Referring to Fig. 6, we assign the $z$ direction to be perpendicular to the surface, the $x$ direction to be parallel to [ $\overline{1} 10]$, and the $y$ direction to be parallel to [001]. For our calculations of the surface Debye temperatures, we have used 100 evenly spaced points in the surface Brillouin zone.

There are several simplifications that we have employed in our present calculations of the surface Debye temperatures. First, since relaxations for the (110) alkali-metal surface layers are experimentally ${ }^{32}$ and theoretically ${ }^{33,34}$ very small (typically $1 \%$ or less), and there is no evidence for surface reconstruction [except for a possible shear displacement of the $K(110)$ surface, ${ }^{35}$ see the discussion below], we have simply used a truncated-bulk model for the equilibrium atomic positions. Second, since the EAM model is based on empirical bulk parameters, any changes in force constants due to the different electronic structure at the surface may not be adequately addressed, although it has been shown for

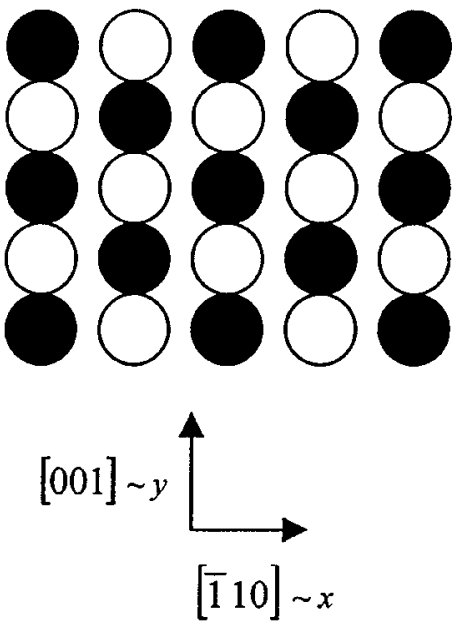

FIG. 6. Schematic of bcc(110) surface illustrating coordinate system used in text. Solid circles, first-layer atoms; open circles, second-layer atoms.

$\mathrm{Cu}(100)$ that a bulk-derived EAM theory can accurately describe surface phonons. ${ }^{36}$ As a point of comparison with $a b$ initio calculations, we have calculated force constants for uniform displacement of a $\mathrm{K}(110)$ surface layer in the $x, y$, and $z$ directions. The $a b$ initio results are, respectively, $0.170,1.63$, and $4.20 \mathrm{~N} / \mathrm{m}$ (Ref. 34), compared to our EAM values of $0.266,1.81$, and $4.04 \mathrm{~N} / \mathrm{m}$. The third simplification, which is likely the most severe, is that we have used a model in which only the first three layers (surface, first underlayer, and second underlayer) are treated dynamically. Deeperlying atoms are fixed at their equilibrium positions. We are currently working to include more dynamical layers in the calculation; therefore the present results should be treated as somewhat preliminary. In spite of this last simplification, the energies and polarizations of our surface modes compare well enough with another EAM calculation ${ }^{37}$ of $\mathrm{Li}(110)$ and $\mathrm{Na}(110)$ surface phonon modes that it appears unlikely our results for the surface Debye temperatures will change by more than $\sim 10 \%$ when more dynamical layers are included.

Our results for the directional Debye temperatures, as well as the average surface Debye temperature $\theta_{D \text {,surf }}$ (which is determined from $\left\langle u^{2}\right\rangle=\left\langle x^{2}+y^{2}+z^{2}\right\rangle$ ), are shown in Table II. For all three alkali metals, the average surface Debye temperature is approximately $10 \%$ less than the bulk Debye temperature, in good qualitative agreement with the results extracted from the Li core-level data. The perpendicular Debye temperature $\theta_{D, z}$ for each metal is significantly lower than the two parallel Debye temperatures, which are both much closer to the bulk Debye temperature.

It is interesting to compare our results with an early theoretical estimate of surface Debye temperatures for $\mathrm{Na}(110) .{ }^{38}$ In that calculation the cohesive energy of $\mathrm{Na}$ was

TABLE II. EAM model determined Debye temperatures.

\begin{tabular}{cccccc}
\hline \hline Alkali metal & $\theta_{D, \text { bulk }}$ & $\theta_{D, \text { surf }}$ & $\theta_{D, x}$ & $\theta_{D, y}$ & $\theta_{D, z}$ \\
\hline $\mathrm{Li}$ & 336 & 295 & 328 & 311 & 261 \\
$\mathrm{Na}$ & 138 & 122 & 137 & 129 & 107 \\
$\mathrm{~K}$ & 93 & 78 & 89 & 82 & 68 \\
\hline \hline
\end{tabular}


TABLE III. Phonon broadening parameters for $\mathrm{Na} 2 p$ and $\mathrm{K} 3 p$ core levels. Parameters were obtained from fitting data with Eq. (1) with Debye temperatures from Table II.

\begin{tabular}{cccccc}
\hline \hline & \multicolumn{2}{c}{ Bulk } & \multicolumn{2}{c}{ Surface } \\
\cline { 2 - 5 } \cline { 5 - 5 } Alkali metal & $G(0)(\mathrm{meV})$ & $C\left(10^{-3} \mathrm{eV}^{3}\right)$ & & $G(0)(\mathrm{meV})$ & $C\left(10^{-3} \mathrm{eV}^{3}\right)$ \\
\hline \multirow{2}{*}{$\mathrm{Na}$} & 50 & 0.03 & 79 & 0.07 \\
$\mathrm{~K}$ & 35 & 0.01 & 56 & 0.02 \\
\hline \hline
\end{tabular}

modeled solely with a simple two-body Morse potential. Bulk and surface Debye temperatures were estimated by treating only a single atom (either at the surface or in the bulk) dynamically and letting $\theta_{D}=\left(\hbar / k_{B}\right) \omega_{0}$, where $\omega_{0}$ is the (angular) oscillation frequency for motion of that atom along a specific direction. The results of that calculation are $\theta_{D, \text { bulk }}=160 \mathrm{~K}, \theta_{D, x}=168 \mathrm{~K}, \theta_{D, y}=149 \mathrm{~K}$, and $\theta_{D, z}$ $=51 \mathrm{~K} .{ }^{38}$ This simple Einstein-oscillator estimate does a reasonably good job of predicting the bulk Debye temperature. Also, the relative ordering of the magnitudes of the surface Debye temperatures predicted with this earlier calculation and our current calculation (see Table II) is the same. In fact, making the same Einstein-oscillator estimate with the present EAM model yields $x, y$, and $z$ surface Debye temperatures of 124,112 , and $89 \mathrm{~K}$, respectively, also in the same relative order as our more sophisticated calculation.

There is one experimental measurement of a surface Debye temperature that can be compared with our calculations. From the temperature-dependent intensity of the specular beam in a LEED study, the effective perpendicular Debye temperature of the $\mathrm{Na}(110)$ surface was determined to be $110 \pm 5 \mathrm{~K}^{32}$ Since the electron mean free path is $\sim 4 \AA$ at the electron kinetic energies used in the LEED study, ${ }^{22}$ this surface Debye temperature is dominated by thermal motion in the first atomic layer and should thus be very close to our calculated value of $\theta_{D, z}=107 \mathrm{~K}$ (see Table II). By taking into account the small contribution from deeper layers, we calculate an effective perpendicular Debye temperature (as measured in the LEED study) of $114 \mathrm{~K}$, in excellent agreement with the experimental result.

The surface Debye temperatures calculated for K(110) are pertinent to prior discussion in the literature regarding a possible shear displacement of the $\mathrm{K}(110)$ surface layer at 25 K. ${ }^{34,35}$ From LEED data it was suggested that the top K layer is uniformly shifted by $0.23 \AA$ along the $x$ direction. ${ }^{35}$ This was followed by an $a b$ initio calculation of first-layersecond-layer force constants for uniform displacement of the first layer. While the calculation found no evidence for a static, shear displacement, it was suggested, based on the very small $x$ direction force constant (refer to the discussion above), that large-amplitude fluctuations along the $x$ direction might be responsible for the LEED observations. By assuming that the $x$-direction motion of each surface atom is governed entirely by the force constant of $0.170 \mathrm{~N} / \mathrm{m}$, an $\mathrm{rms}$ fluctuation $\left\langle x^{2}\right\rangle^{1 / 2}=0.45 \AA$ at $25 \mathrm{~K}$ was estimated. ${ }^{34}$ With the current calculation, however, we can make a much better estimate of the mean-squared displacement. Using the exact form of Eq. (10),

$$
\left\langle x^{2}\right\rangle=\frac{\hbar}{N M} \sum_{n=1}^{3 N} \frac{\hat{x}_{n}^{2}}{\Omega_{n}}\left[\frac{1}{2}+\left(e^{\hbar \Omega_{n} / k_{B} T}-1\right)^{-1}\right],
$$

which is valid at all temperatures, we calculate $\left\langle x^{2}\right\rangle^{1 / 2}$ $=0.12 \AA$ at $25 \mathrm{~K}$, which is much smaller than the earlier estimate. That our calculated fluctuation is much smaller than the force-constant estimate is not surprising given the $x$-direction Debye temperature of $89 \mathrm{~K}$, which is very close to the bulk Debye temperature. Note that this relatively small displacement is not due to the somewhat larger EAM $x$-direction force constant of $0.266 \mathrm{~N} / \mathrm{m}$, which produces $\left\langle x^{2}\right\rangle^{1 / 2}=0.37 \AA$ using the same estimate as in Ref. 34, but is the result of including vibrational modes throughout the surface Brillouin zone. Our results suggest that the proposed shear displacement should be investigated further.

With our values for the bulk and surface Debye temperatures of $\mathrm{Na}(110)$ and $\mathrm{K}(110)$, we are now afforded a somewhat more critical examination of phonon broadening in these two metals. ${ }^{8,9}$ Using our EAM calculated Debye temperatures (see Table II), we have reanalyzed the data from these two systems and have obtained values for $G(0)$ and $C$, which are listed in Table III. Similar to the results for Li, the surface values of $C$ are approximately twice as large as the bulk values.

\section{SUMMARY}

We have measured core-level broadening data from $\mathrm{Li}(110)$ and have obtained bulk-atom and surface-atom values of the zero-temperature phonon width and the effective Debye temperature, which governs the temperature dependence of the broadening. Our results illustrate the importance of obtaining high-quality data in the region below the Debye temperature in order to independently extract these phononbroadening parameters. For comparison purposes, an EAM model ${ }^{20}$ has been used to calculate bulk and preliminary surface Debye temperatures for $\mathrm{Li}(110), \mathrm{Na}(110)$, and $\mathrm{K}(110)$. The relative magnitudes of the bulk and surface Debye temperatures calculated with the EAM model compare favorably with those governing the phonon widths. For the alkali metals, the constant $C$ in the phonon-broadening theory of Hedin and Rosengren ${ }^{3}$ is substantially different in the bulk and at the surface. Theoretical calculations of the parameter $C$ at the surfaces of the alkali metals are the next logical step in understanding surface core-level broadening in these systems.

\section{ACKNOWLEDGMENTS}

The National Synchrotron Light Source at Brookhaven National Laboratory is supported by the U.S. Department of Energy, Division of Materials Sciences and Division of Chemical Sciences. 
${ }^{1}$ See, for example, L. G. Parratt, Rev. Mod. Phys. 31, 616 (1959).

${ }^{2}$ A. W. Overhauser (unpublished); see Ref. 3.

${ }^{3}$ L. Hedin and A. Rosengren, J. Phys. F 7, 1339 (1977).

${ }^{4}$ B. Bergersen, T. McMullen, and J. P. Carbotte, Can. J. Phys. 49, 3155 (1971).

${ }^{5}$ C.-O. Almbladh and A. L. Morales, J. Phys. F 15, 991 (1985).

${ }^{6}$ P. H. Citrin, G. K. Wertheim, and Y. Baer, Phys. Rev. B 16, 4256 (1977).

${ }^{7}$ P. H. Citrin, G. K. Wertheim, and M. Schlüter, Phys. Rev. B 20, 3067 (1979).

${ }^{8}$ D. M. Riffe, G. K. Wertheim, and P. H. Citrin, Phys. Rev. Lett. 67, 116 (1991).

${ }^{9}$ G. K. Wertheim, D. M. Riffe, and P. H. Citrin, Phys. Rev. B 49, 2277 (1994).

${ }^{10}$ W. Theis and K. Horn, Phys. Rev. B 47, 16060 (1993).

${ }^{11}$ E. Weschke and G. Kaindl, J. Electron Spectrosc. Relat. Phenom. 75, 233 (1995).

${ }^{12}$ E. Weschke, A. Höhr, S. Vandré, C. Schüßler-Langeheine, F. Bødker, and G. Kaindl, J. Electron Spectrosc. Relat. Phenom. 76, 571 (1995).

${ }^{13}$ G. K. Wertheim and D. N. E. Buchanan, Phys. Rev. B 43, 13815 (1991).

${ }^{14}$ L. I. Johansson, P.-A. Glans, and T. Balasubramanian, Phys. Rev. B 58, 3621 (1998)

${ }^{15}$ D. M. Riffe and G. K. Wertheim, Phys. Rev. B 47, 6672 (1993).

${ }^{16}$ D. M. Riffe, W. Hale, B. Kim, and J. L. Erskine, Phys. Rev. B 51, 11012 (1995).

${ }^{17}$ D. M. Riffe, G. K. Wertheim, and P. H. Citrin, Phys. Rev. Lett. 63, 1976 (1989).

${ }^{18}$ G. K. Wertheim, P. H. Citrin, and J. F. van der Veen, Phys. Rev. B 30, 4343 (1984).

${ }^{19}$ N. F. Mott and H. Jones, The Theory of the Properties of Metals and Alloys (Dover, New York, 1958), pp. 10-14; C. Kittel, Introduction to Solid State Physics, 7th ed. (Wiley, New York, 1996), p. 126; N. W. Ashcroft and N. D. Mermin, Solid State Physics (Saunders College, Philadelphia, 1976), p. 461.

${ }^{20}$ Y. R. Wang and D. B. Boercker, J. Appl. Phys. 78, 122 (1995).

${ }^{21}$ At a photon energy of $100 \mathrm{eV}$, the $\mathrm{Ni} 3 d$ photoemission cross section is $\sim 350$ times stronger than the Li $2 s$ cross section [J. J. Yeh and I. Lindau, At. Data Nucl. Data Tables 32, 1 (1985)]. Hence, the Ni valence-band spectrum is not obscured by photoemission from the Li conduction band.
${ }^{22}$ G. K. Wertheim, D. M. Riffe, N. V. Smith, and P. H. Citrin, Phys. Rev. B 46, 1955 (1992).

${ }^{23}$ D. M. Riffe, G. K. Wertheim, D. N. E. Buchanan, and P. H. Citrin, Phys. Rev. B 45, 6216 (1992).

${ }^{24}$ S. Doniach and M. Šunjić, J. Phys. C 3, 285 (1970).

${ }^{25}$ It has been suggested by C.-O. Almbladh and P. Minnhagen, Phys. Status Solidi B 85, 135 (1978), that thermal excitation of the electron gas also contributes an amount $\frac{8}{3} \ln (2) \alpha\left(k_{B} T\right)^{2}$ to the square of the Gaussian width. We have not included this contribution in our analysis of the temperature dependence of the phonon broadening. If included, this contribution would alter the numerical values of our extracted values of $\theta_{D}$ by less than $4 \%$. Experimentally, such a contribution is yet to be verified.

${ }^{26}$ G. K. Wertheim, D. M. Riffe, and P. H. Citrin, Phys. Rev. B 49, 4834 (1994).

${ }^{27}$ G. K. Wertheim, D. M. Riffe, and P. H. Citrin, Phys. Rev. B 45, 8703 (1992).

${ }^{28}$ R. A. Johnson and D. J. Oh, J. Mater. Res. 4, 1195 (1989); A. M. Guellil and J. B. Adams, ibid. 7, 639 (1992); M. I. Baskes, Phys. Rev. B 46, 2727 (1992); O. Yifang, Z. Bangwei, L. Shuzhi, and J. Zhangpeng, Z. Phys. B 101, 161 (1996); S. Chantasiriwan and F. Milstein, Phys. Rev. B 58, 5996 (1998).

${ }^{29}$ H. G. Smith, G. Dolling, R. M. Nicklow, P. R. Vijaraghavan, and M. K. Wilkinson, Symposium on Neutron Inelastic Scattering (IAEA, Vienna, 1968), p. 149.

${ }^{30}$ A. D. B. Woods, B. N. Brockhouse, R. H. March, A. T. Stewart, and R. Bowers, Phys. Rev. 128, 1112 (1962).

${ }^{31}$ R. A. Cowley, A. D. B. Woods, and G. Dolling, Phys. Rev. 150, 487 (1966).

${ }^{32}$ S. Andersson, J. B. Pendry, and P. M. Echenique, Surf. Sci. 65, 539 (1977).

${ }^{33}$ R. Rodach, K. P. Bohnen, and K. M. Ho, Surf. Sci. 209, 481 (1989).

${ }^{34}$ A. F. Wright and D. C. Chrzan, Phys. Rev. Lett. 70, 1964 (1993).

${ }^{35}$ B. S. Itchkawitz, A. P. Baddorf, H. L. Davis, and E. W. Plummer, Phys. Rev. Lett. 68, 2488 (1992).

${ }^{36}$ J. S. Nelson, E. C. Sowa, and M. S. Daw, Phys. Rev. Lett. 61, 1977 (1988).

${ }^{37}$ I. Y. Sklyadneva, A. V. Berch, and E. V. Chulkov, Fiz. Tverd. Tela (Leningrad) 37, 2646 (1995) [Phys. Solid State 37, 1454 (1995)].

${ }^{38}$ D. P. Jackson, Surf. Sci. 43, 431 (1974). 